\begin{tabular}{|c|l|}
\hline Title & Random dynamical systems modeling for brain wave synchrony \\
\hline Author(s) & Kazuhiro, Matsumoto; Y uzuru, Sato; Hiroshi, Endo; Keiichi, Kitajo \\
\hline Citation & Hokkaido University Preprint Series in Mathematics, 1037, [1] \\
\hline Issue Date & 20136-27 \\
\hline DOI & 10.14943/84181 \\
\hline Doc URL & http://hdl.handle.net/2115/69841 \\
\hline Type & bulletin (article) \\
\hline File Information & pre1037.pdf \\
\hline
\end{tabular}

Instructions for use 


\title{
Random dynamical systems modeling for brain wave synchrony
}

\author{
Kazuhiro Matsumoto*, Yuzuru Sato ${ }^{\dagger}$, Hiroshi Endo ${ }^{\ddagger \S}$, and Keiichi Kitajo ${ }^{\S}$ \\ ${ }^{*}$ Research \& Development Section, Yamaha Motor Co., Ltd. \\ 2500 Shingai, Iwata, Shizuoka 438-8501, Japan \\ $\dagger$ RIES / Department of Mathematics, Hokkaido University \\ Kita 12 Nishi 6, Kita-ku, Sapporo, Hokkaido 060-0812, Japan \\ $\ddagger$ Graduate School of Creative Science and Engineering, Waseda University \\ 3-4-1, Okubo, Shinjuku-ku, Tokyo 169-8555 \\ §RIKEN Brain Science Institute \\ 2-1 Hirosawa, Wako-shi, Saitama 351-0198, Japan \\ Email: matsumotokazuhi@yamaha-motor.co.jp, ysato@math.sci.hokudai.ac.jp, \\ endo-hiroshi@brain.riken.jp, kkitajo@brain.riken.jp
}

\begin{abstract}
A random dynamical systems model is studied to understand coupled dynamics of auditory area and motor area modulated by external force. We measure transfer entropy of coupled oscillators with the presence of noise to explain results of human brain wave experiments.
\end{abstract}

\section{Introduction}

Why does one feel excited when listening to music? To consider this problem, we investigate dynamics within our brain when emotional shifts occur associated with music perception. This study is also motivated to develop products based on interaction between human motion and emotional response at Yamaha Motor Co., Ltd.

It has been reported that interactions between auditory area and motor area are involved in musical processing in human brain [11]. However, there are very few studies on causal relationship between the two areas associated with music perception. We therefore investigate this problem by measuring transfer entropy, which is an alternative entropy measure to mutual information taking information transport into account [1], in large-scale neural synchrony networks mediating music perception by human brain wave experiments.

Physiological data such as electroencephalography (EEG: brain wave), heartbeat and respiration have been studied by using oscillatory systems with coupled oscillators $[2,3,4,5,8]$. Lin et al. investigate the influence of random external force to a coupled phase oscillator system and discover shear induced chaos [6], which is explained in terms of random strange attractors. Sato et al. study a random sine circle map to study synchrony between human heartbeats and pedaling rhythms with music as the external modulation [7]. Inspired by these studies, we adopt randomly perturbed coupled oscillators to model EEG synchrony associated with music perception. We then examine the transfer entropy both in experimental and modeling data.

\section{Experimental data}

Fourteen healthy right-handed volunteers participated in the music perception experiment. All subjects gave written informed consent prior to participation in this study. The study was approved by the RIKEN Ethical Committee. In our experimental setting, there were two conditions: (1) resting (without music) and (2) listening to music. Each condition lasted for three minutes. We measured 63-channel with a sampling rate of 1,000 Hz. Figure 1 shows an experimental view. The music used in our experiments was Born This Way by Lady Gaga.

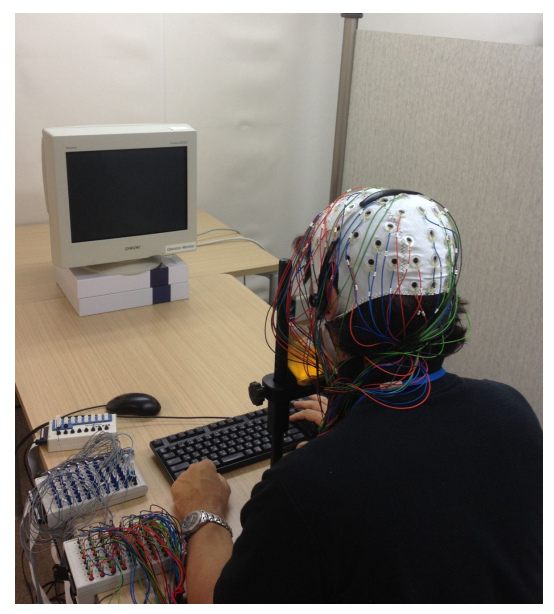

Figure 1: Experimental setup.

We extracted instantaneous phase of EEG signals and analyzed phase dynamics $[9,10]$. In our preliminary analyses we found most prominent large-scale neural synchrony associated with music perception at $8 \mathrm{~Hz}$. We therefore analyzed information transfer between $8 \mathrm{~Hz}$ phase signals from auditory area (electrode T7) and motor area (electrode C3) assuming that these areas are involved in music perception [11]. Figure 2 shows typical EEG phase time series data from a single subject for electrode $\mathrm{T} 7$ and $\mathrm{C} 3$ at $8 \mathrm{~Hz}$. 

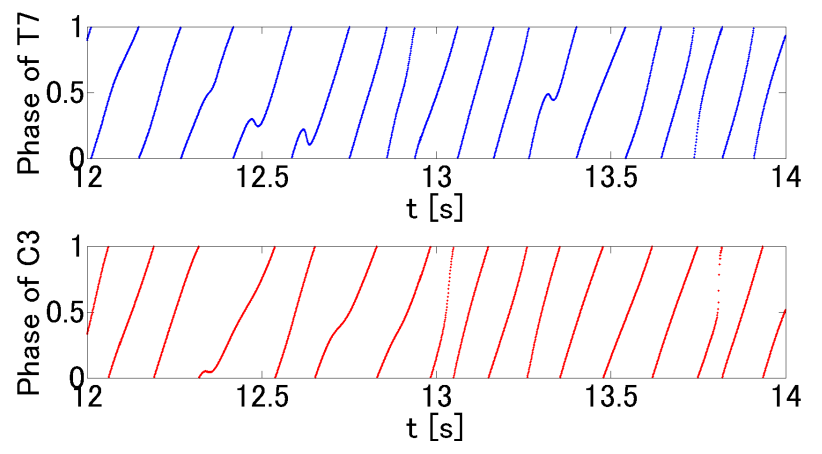

Figure 2: Top: a time series of the phase for T7 (auditory). Bottom: a time series of the phase for $\mathrm{C} 3$ (motor).

The phases of T7 (auditory) and C3 (motor) are described with variables $\theta_{1}$ and $\theta_{2}$, respectively. The transfer entropy from the auditory area to the motor area for length $N$ time series data with resolution $\Delta t$ is represented as

$T_{1 \rightarrow 2}(\tau)=\sum_{n=0}^{N} P\left(\theta_{2, n \Delta t+\tau}, \theta_{2, n \Delta t}, \theta_{1, n \Delta t}\right) \log \frac{P\left(\theta_{2, n \Delta t+\tau} \mid \theta_{2, n \Delta t}, \theta_{1, n \Delta t}\right)}{P\left(\theta_{2, n \Delta t+\tau} \mid \theta_{2, n \Delta t}\right)}$.

The transfer entropy for the other direction $T_{2 \rightarrow 1}(\tau)$ is defined similarly.

Figure 3 shows the transfer entropies between $\mathrm{T} 7$ and C3 for the same subject as in Fig. 2, where $\tau=0.125$ [s] $=$ $\frac{1}{8[\mathrm{~Hz}]}$ under the experimental conditions with and without music . Note that $T_{2 \rightarrow 1}$ becomes greater than $T_{1 \rightarrow 2}$ under the condition with music. This difference is statistically significant over the 14 subjects $(P<0.05)$. Therefore, the transfer entropy from the motor area to the auditory area is rather dominant in this case.

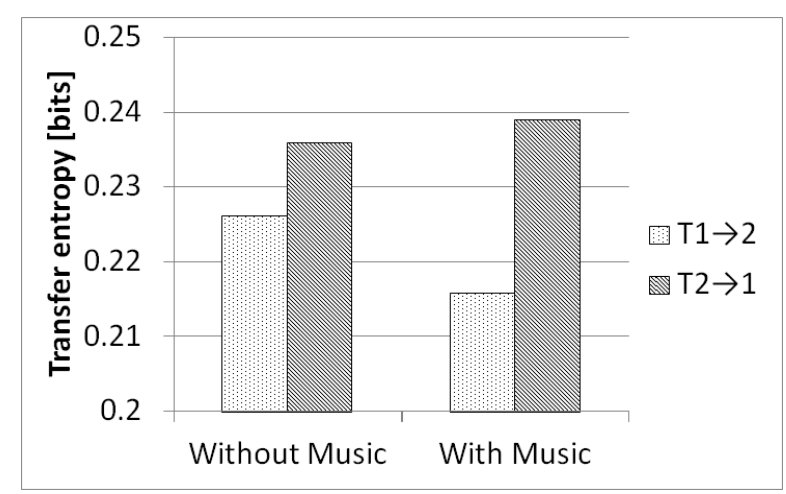

Figure 3: Transfer entropy of experimental data.

\section{Model simulations}

Starting with coupled oscillators as a model of phases dynamics of EEG in the auditory area and the motor area, we introduce a common noise term to describe interaction with other brain areas, which is a white Gaussian random variable $\xi$. Regarding the effect of music as a random external force $I$ following a white Gaussian distribution, we have the following model on the torus $\mathbb{T}^{2}=[0,1) \times[0,1)$.

$$
\begin{aligned}
\dot{\theta}_{1} & =\omega+\frac{c_{21}}{2 \pi} \sin \left(2 \pi\left(\theta_{2}-\theta_{1}\right)\right)+\frac{\xi}{2 \pi} \sin \left(2 \pi \theta_{1}\right) \\
& +\frac{I}{2 \pi} \sin \left(2 \pi \theta_{1}\right) \\
\dot{\theta}_{2} & =\omega+\frac{c_{12}}{2 \pi} \sin \left(2 \pi\left(\theta_{1}-\theta_{2}\right)\right)+\frac{\xi}{2 \pi} \sin \left(2 \pi \theta_{2}\right)
\end{aligned}
$$

where $\omega$ is the natural frequency of phase oscillators, and $c_{12}$ and $c_{21}$ are the coupling strengths from $\theta_{1}$ to $\theta_{2}$ and from $\theta_{2}$ to $\theta_{1}$, respectively.

The natural frequency is normalized to be $\omega=1$, corresponding to $8 \mathrm{~Hz}$ frequency filtering of experimental data. Both coupling strengths are supposed to be weak and identical, and set to be $c_{12}=c_{21}=0.1$. The influence of common noise is supposed to be relatively strong and steady. The mean of $\xi$ is 0 and the variance of it is 0.5 . These parameters are set to be constant throughout the model simulations. We are interested in the influence of external force to the system, which is regarded as an open and uncertain environment for the coupled oscillators. We assume that the external force $I$ follows a white Gaussian distribution with mean 0.1 , and its variance $\sigma$ is a control parameter. The open and uncertain environment is modeled as the external noise with fluctuation size $\sigma$. In particular, the condition without music is modeled by setting $\sigma=0$, and the condition with music by $\sigma \sim 2.5$.

Figure 4 and 5 show the time series with $\sigma=0$ and with $\sigma=2.5$ respectively, which are generated with a randomly chosen initial condition and an external noise realization.
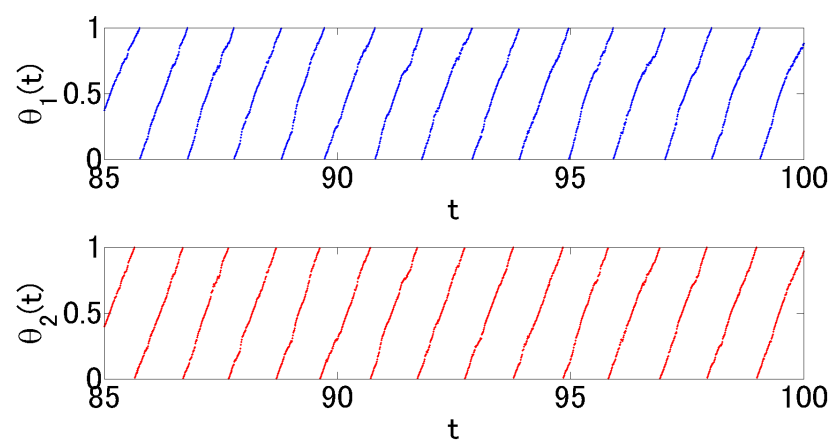

Figure 4: Time series with $\sigma=0$. Top: $\theta_{1}(t)$. Bottom: $\theta_{2}(t)$

One can see that the common noise causes phase modulation in both oscillators even with $\sigma=0$ in Fig. 4. When the external noise becomes stronger, at $\sigma=2.5$ as in Fig. 5 , perturbation to the oscillator $\theta_{1}$ weakly propagates to the oscillator $\theta_{2}$.

In order to compare with the experimental data, we calculate the transfer entropy in our model. The transfer entropy at $\tau=1$ for $\sigma \in[0,10]$ is depicted in Fig. 6, where 

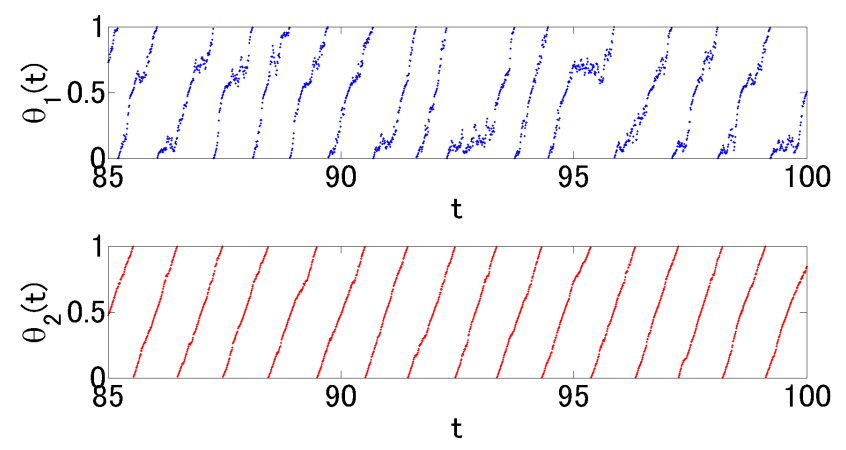

Figure 5: Time series with $\sigma=2.5$. Top: $\theta_{1}(t)$. Bottom: $\theta_{2}(t)$.

the calculation is realized by taking an average of transfer entropies over 20 sets of randomly chosen initial condition and noise realization for each $\sigma$. Notably, it turns out that

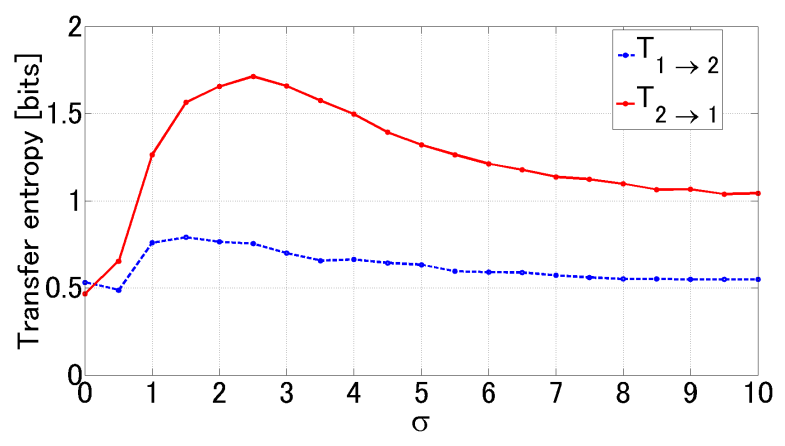

Figure 6: Transfer entropy with $\sigma \in[0,10]$ in the model. Dotted line: $T_{1 \rightarrow 2}$. Solid line: $T_{2 \rightarrow 1}$.

$T_{2 \rightarrow 1}$ becomes greater than $T_{1 \rightarrow 2}$ at $\sigma \sim 2.5$. This result is comparable with the experimental results.

How can we interpret the behavior of transfer entropy? It would be useful to observe phase portraits to understand it. Figure 7 shows the phase portraits for $\sigma=0,2.5$ and 6, together with a deterministic limit cycle in the case that the random variable $\xi$ vanishes and $\sigma=0$.

In general, transfer entropy between two independent random variables is 0 . To the contrary, for totally predictable dynamics without irregularity, such as oscillation, transfer entropy is 0 again. In our model, when $\sigma$ is around 0 , the trajectories still stay close to the deterministic limit cycle. This means that both $\theta_{1}$ and $\theta_{2}$ keep small predictability under presence of common noise. Thus, both $T_{1 \rightarrow 2}$ and $T_{2 \rightarrow 1}$ remain small, but not 0 . However, when $\sigma$ becomes larger around $\sigma=2.5, \theta_{1}$ becomes unpredictable because of the external noise, while $\theta_{2}$ keeps its predictability because of the weak coupling. As a result, only $T_{2 \rightarrow 1}$ increases because transfer entropy from a predictable variable to an unpredictable variable is larger as long as the variables are still causally dependent. On the other hand, when $\sigma$ becomes very large around $\sigma=6, T_{2 \rightarrow 1}$ decreases again, as $\theta_{1}$ becomes an effectively independent variable from $\theta_{2}$.
With $\sigma=2.5$, the trajectories are perturbed by the external noise but still cope with the deterministic structure in state space including the limit cycle and its basins of attraction. However, with $\sigma=6$ trajectories show almost random behavior independent of the deterministic state space structure. In this case, transfer entropy becomes smaller again, but not 0 , because of density concentration to $\theta_{1}=0$ and to $\theta_{1}=0.5$ based on the random variable $I$ multiplicative to $\sin 2 \pi \theta_{1}$. This can be one of the mechanisms of the observed noise-induced transition of transfer entropy and the reason why a peak exists in $T_{2 \rightarrow 1}$ curve at a point around $\sigma=2.5$.
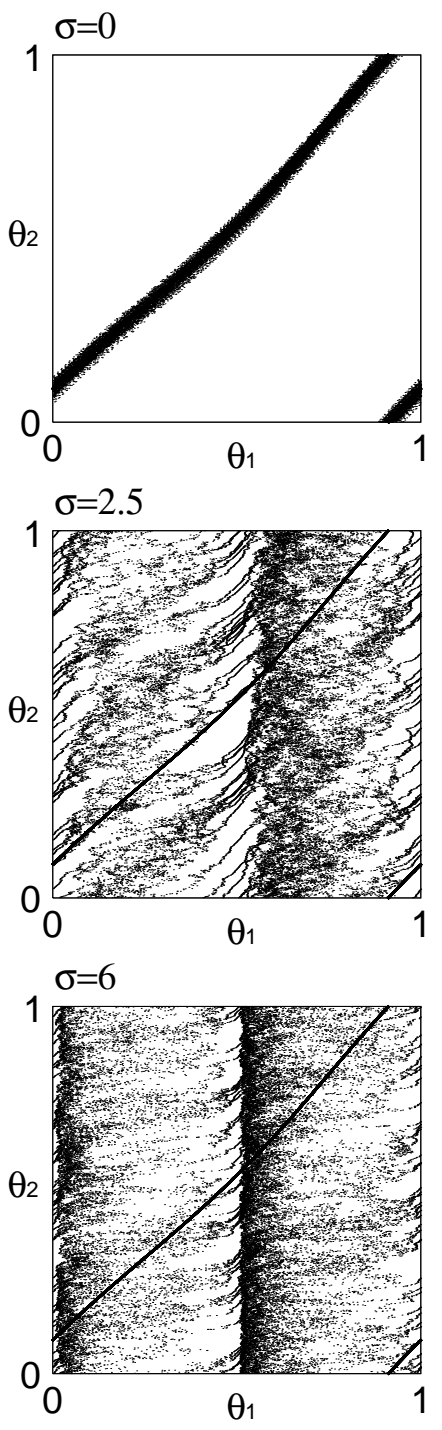

Figure 7: Phase portrait for each $\sigma$. A typical trajectory of Eqs. (1) and (2) is depicted with a randomly chosen initial condition and a noise realization by using Euler-Maruyama scheme. Parameters are set to (top) $\sigma=0$, (middle) $\sigma=$ 2.5, and (bottom) $\sigma=6$. For reference, a deterministic limit cycle in the case that the random variable $\xi$ vanishes and $\sigma=0$, is superimposed as a solid line in each phase portrait. 
In all cases with parameters $\sigma=0,2.5,6$, we eventually observe noise-induced synchronization for an ensemble of initial conditions. However, we can say that dynamics with $\sigma=2.5$ shown in Fig. 7 is the most complex because it is semi-predictable while dynamics with $\sigma=0$ is almost predictable and those with $\sigma=6$ is almost unpredictable. It is thought that this simple model may explain our experimental results of transition of transfer entropy.

Considering further about transfer entropy under presence of noise-induced phenomena in nonlinear dynamics, such as stochastic resonance [12], noise-induced synchronization [13], or noise-induced chaos [14], external noise typically alters predictability of observed systems. In some cases, large noise rather enhances predictability, but in other cases, small noise causes significant unpredictability. Thus, one can find that, in general, it is far from trivial to compute transfer entropy in noised dynamical systems, apart from the problem whether causality is measurable.

\section{Conclusions and Discussions}

We investigated the effect of music from the viewpoint of brain wave synchrony in auditory-motor interactions. We extracted the time series of phase from EEG data and calculated the information flow by using the transfer entropy. As a result, we confirmed that transfer entropy from the motor area to the auditory area becomes greater than those with reversed direction under the condition with music. We constructed a model with two randomly coupled phase oscillators to explain the experimental data. The characteristics of this model is to have two stochastic terms: One is the external noise which expresses an uncertain input such as music, the other is the internal noise which expresses interaction with other brain areas. The behavior of transfer entropy is consistent with the experimental results.

In our experiments, transfer entropy from the motor area to the auditory area becomes dominant by the effect of music. Yet one might think that it looks contradictory, because the upstream auditory area receiving music is supposed to drive the downstream motor area. In this sense, music enables the direction of dominant transfer entropy to be reversed, which may imply that information transport from humans to external environments is measured and observed with this entropy measure. Going back to the original motivation of this study, we would like to develop exciting products which lead to dynamic change of information flow.

\section{Acknowledgments}

This work was supported by a collaborative research grant from Yamaha Motor Co., Ltd.

\section{References}

[1] T. Schreiber, "Measuring information transfer," Phys. Rev. Lett., 85, p461-464, (2000).
[2] Y. Kuramoto, "Chemical Oscillations, Waves, and Turbulence,” Springer-Verlag, Berlin, (1984).

[3] L. Glass, M. R. Guevara, A. Shrier, and R. Perez, "Bifurcation and Chaos in a Periodically Stimulated Cardiac Oscillator," Physica, 7D, p89-101, (1983).

[4] L. Glass and M.C. Mackey, "From clocks to chaos: the rhythms of life," Princeton Univ. Press, Princeton, NJ, (1988).

[5] M. Courtemanche, L. Glass, J. Bi Lair, D. Scaglioti'i, and D. Gordon, "A circle map in a human heart," Physica, D40, p299-310, (1989).

[6] K. Lin and L.-S. Young, "Shear-induced chaos," Nonlinearity, 21 p899-922, (2008).

[7] Y. Sato and K. Matsumoto, "Random dynamics from time-serieses of physiological rhythms," Hokkaido University Preprint Series in Mathematics, \#1012, (2012).

[8] D. Taylor and P. Holmes, "Simple models for excitable and oscillatory neural networks," J. Math. Biol., 37, p419-446, (1997).

[9] K. Kitajo, S. M. Doesburg, K. Yamanaka, D. Nozaki, L. M. Ward, and Y. Yamamoto,"Noise-induced largescale phase synchronization of human brain activity associated with behavioural stochastic resonance." Europhysics Letters, 80 p40009-1-6, (2007).

[10] F. Varela, J.P. Lachaux, E. Rodriguez, and J. Martinerie, "The brainweb: phase synchronization and large-scale integration," Nature Review Neuroscience, 2, p229-239, (2001).

[11] R. J. Zatorre, J. L. Chen, and V. B. Penhune, "When the brain plays music: auditory-motor interactions in music perception and production," Nat Rev Neurosci, 8 (7), p547-58, (2007).

[12] K Kitajo, D. Nozaki, L. M. Ward, and Y. Yamamoto, "Behavioral stochastic resonance within the human brain," Phys. Rev. Lett. 90, p218103, (2003).

[13] J. Teramae and D. Tanaka, "Robustness of the noiseinduced phase synchronization in a general class of limit cycle oscillators," Phys. Rev. Lett., 93, p204103, (2004).

[14] G. Mayer-Kress and H. Haken, "The influence of noise on the logistic model," Journal of Statistical Physics, 26, p149-171, (1981). 